\title{
Multi-Scale Adaptive NICK Thresholding Method for ALPR system
}

\author{
Rohollah Mazrae Khoshki ${ }^{1}$, Subramaniam Ganesan ${ }^{1}$ \\ Electrical and Computer Engineering Department, Oakland University, Rochester, MI, USA ${ }^{1}$
}

\begin{abstract}
This paper presents improved thresholding method called "Multi-Scale Adaptive NICK thresholding method" for Automatic License Plate Recognition (ALPR) System. By applying Connected Components Labelling (CCL) algorithm on the Binary image, license plate characters candidates will be determined. Improved thresholding method is vital to approach all the license plate characters on the binary image otherwise we might lose license plate characters under various illumination conditions and different weather conditions like rain, snow and fog. Most advantages of this method in compare with Niblack and Savola thresholding method is that it could be adapted for both types of degradation: interfering degradation and intensity degradation depends based on scale or window size, therefore you can improve all types of degradation. The interfering degradation will be restored on high scales size to cover sufficient data from the original input imag. In compare, intensity degradation needs very small scales to deal with disjoint and broken characters in the license plate. In this method we don't miss any object in the binary image as license plate character's candidate.
\end{abstract}

Keywords: ALPR (Automatic License Plate Recognition) system, NICK thresholding method, Connected Components Labelling (CCL), Savola method, Niblack method.

\section{INTRODUCTION}

The license plate numbers define a legal certificate of acceptable and improved images without knowledge about vehicles to driven in the roads and public traffic. All the source of degradation. For connected components vehicles in the world must have their license id included labelling Thresholding play an important role. In this letters and/or numbers, written on a license plate and paper we introduced improved Multi-Scale Adaptive mounted onto vehicle's body, some country and states Thresholding Method applied on ALPR system. mounted on front and rear sides and some at least at the rear side. Therefore vehicle without properly mounted, clearly visible and readable license plate cannot drive on the roads and highways. Managing and controlling all the vehicles in transportation system is very hard and time consuming especially in big and high traffic cities. Intelligent Transportation System (ITS) helps us by managing the cameras on the road to detecting the vehicles based on their license plate.

Automatic License Plate Recognition (ALPR) systems find the vehicle license plate from images taken by camera [1-2]. Many research have been done for ALPR systems but improving of ALPR systems based on accuracy and computation time is still on demand. ALPR system should have high degree of reliability in different illumination condition.

Regular previous ALPR system [1] has four main processing stage for recognition of license plate in input images according to:

- Pre-Processing and Image Enhancement

- License plate Location Extraction

- Character Segmentation

- Character Recognition

It is essential to separate objects as character's candidate from backgrounds of license plate and find the best thresholding method for Binarization which will correctly isolate all the connected component from each other even in low contrast areas of the input image, regardless of variable and complex background intensity and noise. When we calculate a threshold, if the pixel value is below the threshold value its value set to 0 (background), otherwise set to 1 (foreground).

We need to evaluate the best thresholding method for Binarization, by investigate over different method for Thresholding, we design improved Multi-Scale adaptive NICK Thresholding method as the best appropriate method. License plate character images require logical content preservation during Thresholding stage, for example, character connectivity must be maintained for optical character recognition. In the following section we review previous work on Thresholding and after that explain proposed method Multi-Scale Adaptive NICK Thresholding in details.

\section{REVIEW OF PREVIOUS WORK ON THRESHOLDING}

Pre-processing and Image enhancement is an initial and Sankur [3], thresholding methods are categorized vital step in most of image processing algorithm, image the data obtain from the input image:

enhancement algorithms propose a wide range of • Histogram-shaped-based approaches for modifying images to achieve visually

- Clustering-based 


\section{International Journal of Advanced Research in Computer and Communication Engineering}

Vol. 4, Issue 10, October 2015

- Entropy-based

- Attribute similarity methods

- Object attribute-based

- Spatial approaches

- Local methods

Generally the thresholding techniques can be categorized in two major groups: global thresholding and local thresholding. Global Binarization such as Otsu [4] and the entropy-based methods [5] try to find a single threshold value for the whole image Binarization. Then each pixel is assigned to foreground or background segment based on its grey value in compare with single global threshold value. For a long time Global Binarization methods [4] work great for typical images that objects intensity are well separated from background intensity, they are fast in compare with local Binarization but they are inappropriate for complex background and degraded images for example non-uniform illumination over the image, so global thresholding for license plate character extraction is not applicable; we might lose some characters in Binarization step. To overcome these situations, local thresholding techniques have been suggested for Binarization.

Locally adaptive thresholding methods could be efficient choice for license plate character detection Binarization stage, in these methods a threshold is calculated at each pixel, the value of the threshold depends on some local statistics like average, variance, and local window size or their combinations. Normally several adjustable parameters are exist in adaptive methods, Deravi and Pal [6] were the pioneers in adaptive techniques for thresholding. One of the famous and pioneer local thresholding methods is Niblack threshold filter [7], it is performed on the degraded image that contains original image and noise. Local Niblack filter depends on two statistical measurement the local mean $\mu(x, y)$ and local variance $\delta(\mathrm{x}, \mathrm{y})$ of $\mathrm{M} \mathrm{x} N$ window size block centered at pixel $(\mathrm{x}, \mathrm{y})$ location in an image. The method calculates threshold for each pixel by shifting a rectangular window [M N] size across the image. The threshold $\mathrm{T}(\mathrm{x}, \mathrm{y})$ for the pixel $\mathrm{p}$ at centre of the window $[\mathrm{M} \mathrm{N}]$ in the $\mathrm{x}$ row and $\mathrm{y}$ column of input image is presented according to equation (1):

$$
\begin{gathered}
T(x, y)=\mu(x, y)+[k \cdot \delta(x, y)] \\
T(x, y)=\mu(x, y)+K \cdot \sqrt{\frac{1}{N t} \cdot \sum_{i=1}^{n}\left(P_{i}-\mu(x, y)\right)^{2}} \\
\mathrm{~T}(\mathrm{x}, \mathrm{y})=\mu(\mathrm{x}, \mathrm{y})+\mathrm{K} \cdot \sqrt{\frac{\sum_{\mathrm{i}=1}^{\mathrm{n}} \mathrm{P}_{\mathrm{i}}^{2}}{\mathrm{Nt}}-\mu^{2}(\mathrm{x}, \mathrm{y}) \cdot}=\mu(\mathrm{x}, \mathrm{y})+ \\
\mathrm{k} \sqrt{\mathrm{C}}
\end{gathered}
$$

Where $\mathrm{Nt}$ is the number of pixels in the gray image, $\mu$ $(\mathrm{x}, \mathrm{y})$ is the average value of the pixels pi, $\mathrm{K}$ is the bias that controls the level of adaptation varying the threshold value. Negative value of $\mathrm{k}$ is used for input images suffering low-intensity degradation like character parts broken while a positive value of $\mathrm{k}$ helps in the removal of high-intensity degradation like complex backgrounds. Based on experiments results author suggested $\mathrm{k}=-0.2$. This method can intensely identify the characters and objects in foreground. However, it may fail on smooth backgrounds and bad illumination condition; also it creates white noise pixels in the smooth local backgrounds area far from characters and objects.

In the case that the noises are not completely independent of the image content, like additive or multiplicative noise, Adaptive Niblack threshold filters could deal with, these filters efficiently used when there is no noise information in input image data, because instead of global image characteristic, the filtering implemented based on the local characteristics of the image like local mean value and local standard deviation value not globally all over the image. Depending on the applications, some types of adaptive Niblack filters may perform well at removing noise.

In local thresholding method of Bernsen [8] the threshold is set at the mean value of the minimum and maximum of a local window. This algorithm adapted weakly to even small changes in illumination and complex backgrounds. In some methods edge information used along with thresholding. In [15] suggest an algorithm that uses edge data for enhancement of foreground objects related to the neighbourhood's pixels. These methods working good when edge information is completed, edges may be weak and disconnected when the input is a degraded image. Also, strong interfering patterns could produce some false edges information. In case we loosing edge information in some portion of image, it will be affected final thresholding results.

Sauvola thresholding filters [9] improved Niblack limitation in low contrast portion of image. The Sauvola threshold filter is defines according to equation (2):

$$
T(x, y)=\mu(x, y) \cdot\left[1+k \cdot\left(\frac{\delta(x, y)}{R}-1\right)\right]
$$

Where $T(i, j)$ determine threshold at pixel in location row ' $\mathrm{x}$ ' and column ' $\mathrm{y}$ ' in image, $\mu(\mathrm{x}, \mathrm{y})$ and $\delta(\mathrm{x}, \mathrm{y})$ are mean and standard deviation of pixels in the local window size $[\mathrm{m}, \mathrm{n}]$ cantered at pixel $(\mathrm{x}, \mathrm{y})$. $\mathrm{R}$ defines as dynamic range of standard deviation based on experimental results $R=125$ and parameter $\mathrm{k}$ accepts positive values in range $[0.2,0.5]$. The author suggested $\mathrm{k}=0.2$. Positive values for $\mathrm{k}$ allow the method to capture background distributions in small sampling windows which helps to achieve a clean background area far from foreground objects. This filter will be adapted by the local properties and structures of an image.

As we mentioned before the big drawback of Niblack's method is that the output binary image usually has a large amount of white pixels as noise in smooth background especially in areas without character information in image. With Sauvola's method, the background noise problem that appears in Niblack's method will be removed but in many cases where there are less intensity variations and low contrast; characters become extremely thinned and broken and sometimes the characters missed in the output binary image.

Generally, neither of these methods could deal with all the issues. The methods deal well on some problems and fail 
on others. Furthermore, for most of these methods find objects in complex backgrounds is a big challenge. To overcome this limitation, we introduce modified MultiScale Adaptive NICK thresholding method.

\section{III.PROPOSED MULTI-SCALE ADAPTIVE NICK THRESHOLDING METHOD}

Generally, in [10] almost all types of image degradation regarding to character detection divided into two major types: (1) interfering degradation and (2) intensity degradation. Interfering degradation means the presence of any other source of data and information other than the original image data, like noise and bad illumination condition. These interfering sources can contain characters or non-characters forms of information. Complex backgrounds are examples of non-character's candidate interfering degradation, while shadow through characters is related to character's candidate degradation. In other hands, in intensity degradation, some portions of the characters candidate might lose in thresholding stage because it appears with intensity value near to the background.

In this section, first we discuss efficient and fast method to calculate the $\mu(\mathrm{x}, \mathrm{y})$ mean and $\delta(\mathrm{x}, \mathrm{y})$ variance of local windows based on Integral sum image. In this method $\mu$ $(\mathrm{x}, \mathrm{y})$ and $\delta(\mathrm{x}, \mathrm{y})$ are calculated independently of local window size. Then we explain adaptive NICK thresholding method and present Multi-Scale adaptive NICK thresholding method as efficient and accurate method for thresholding degraded image on bad illumination condition or complex background.

\section{A. Integral Sum Image}

Adaptive NICK thresholding method work as local thresholding, main drawbacks of the local thresholding techniques are time consuming and local window size dependency. To achieve a fast local thresholding method for real time operations Bradly [12] introduce the technique of integral sum image to calculate mean and variance of image regardless of local window size that would be efficient for Multi-Scale function for improvement that we will explained later. In this method the local mean could be measured efficiently in a single pass without depending on local window size by integral sum image.

It worth to notice that computation of $\mu(\mathrm{x}, \mathrm{y})$ and $\delta(\mathrm{x}, \mathrm{y})$ for each pixel $(\mathrm{x}, \mathrm{y})$ in a traditional way results in a time complexity of $\mathrm{O}\left(W^{2} \cdot N^{2}\right)$ for an image size $\mathrm{N} \times \mathrm{N}$ and local windows $\mathrm{W} \times \mathrm{W}$, while by using integral sum image to determine local sum the computational time calculated as $\mathrm{O}\left(N^{2}\right)$ regardless of the local window dimension.

An integral sum image 'Ing' of an input image 'In' is defined as the image that intensity at each pixel position $\operatorname{Ing}(x, y)$ is equal to the sum of the intensities of all the pixels above and to the left side of that position $(x, y)$ in the original image, according to equation (3), Integral sum image intensity at $(\mathrm{x}, \mathrm{y})$ calculated as:

$$
\operatorname{Ing}(x, y)=\sum_{i=1}^{x} \sum_{j=1}^{y} \operatorname{In}(i, j)
$$

The integral sum image of gray-scale input image size [m n] could be calculated in single pass rendering of input image according three below steps respectively:

- Calculate integral sum of first row at $(1, y) ; \operatorname{Ing}(1, y)=$ $\operatorname{In}(1, \mathrm{y})+\operatorname{Ing}(1, \mathrm{y}-1), \mathrm{y}=2,3,4, \ldots, \mathrm{n}$

- Calculate integral sum of first column at $(x, 1)$; $\operatorname{Ing}(\mathrm{x}, 1)=\operatorname{In}(\mathrm{x}, 1)+\operatorname{Ing}(\mathrm{x}-1,1), \mathrm{x}=2,3,4, \ldots, \mathrm{m}$

- Calculate integral sum of entire pixels at $(\mathrm{x}, \mathrm{y})$; $\operatorname{Ing}(\mathrm{x}, \mathrm{y})=\operatorname{In}(\mathrm{x}, \mathrm{y})+\operatorname{Ing}(\mathrm{x}, \mathrm{y}-1)+\operatorname{Ing}(\mathrm{x}-1, \mathrm{y})-\operatorname{Ing}(\mathrm{x}-1, \mathrm{y}-$ 1), $\mathrm{x}=2,3,4, \ldots, \mathrm{m}, \mathrm{y}=2,3,4, \ldots, \mathrm{n}$

Figure 1 shows the computation of an integral sum image at these three steps in the simple example; accordingly Ing_w $(x, y)$ the sum of the integral image for any local rectangle window with upper left corner $(\mathrm{x} 1, \mathrm{y} 1)$, and lower right corner $(\mathrm{x} 2, \mathrm{y} 2)$ can be computed in constant time by using integral sum image based on equation (4):

$\operatorname{Ing} \_w(x, y)=\operatorname{Ing}(x 2, y 2)-\operatorname{Ing}(x 2, y 1-1)-\operatorname{Ing}(x 1-1, y 2)+\operatorname{Ing}($

$$
\mathrm{x} 1-1, \mathrm{y} 1-1)
$$

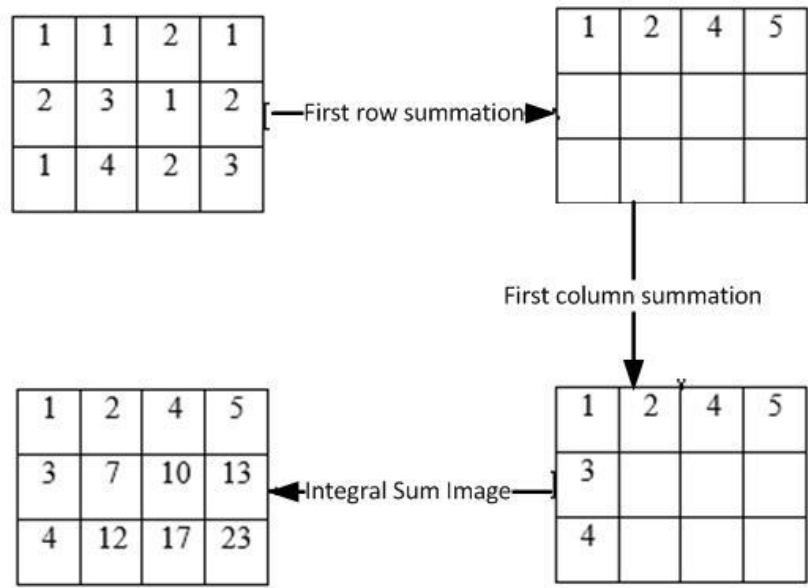

Fig. 1. Step process calculation of Integral Image

Therefore by using Bradly method [12] as initial process for finding local mean and standard deviation of the neighbouring pixels in a window regardless of window size, so we decrease the computation time. This advantage of bradly method makes it perfect for Multi-Scale NICK method thresholding with different windows size scaling that we will explain in next section.

\section{B. Multi-Scale Adaptive NICK thresholding Method}

The problems caused by noise, illumination and other degradations source could be addressed by adaptive NICK thresholding filters [13]. NICK method presents an improvement in the Niblack algorithm; it removes noise as the white pixels in smooth background area by shifting down the Binarization threshold value in smooth background area. It used the Niblack algorithm as fundamental and source method. The NICK thresholding formula is according equation (5):

$$
T(x, y)=\mu(x, y)+K \cdot \sqrt{\frac{\left(\sum_{i=1}^{n} P_{i}{ }^{2}-\mu^{2}(x, y)\right)}{N t}}=\mu(x, y)+
$$

Where, $\mathrm{T}(\mathrm{x}, \mathrm{y})$ is threshold at $\operatorname{pixel}(\mathrm{x}, \mathrm{y}), \mathrm{k}$ is the Niblack factor, $\mu(x, y)$ is mean grey value of local window of size 
$[\mathrm{x}, \mathrm{y}]$ centered at pixel $(\mathrm{x}, \mathrm{y}), \mathrm{pi}$ is pixel value of grey scale image, $\mathrm{Nt}$ is number of total pixels. In equation (6) relation between $\mathrm{C}$ and $\mathrm{D}$ determined by using equation (1) and (5):

$$
\begin{gathered}
C-D=\frac{\sum_{i=1}^{n} P_{i}{ }^{2}}{N t}-\frac{m^{2}(x, y)}{N t}-\frac{\sum_{i=1}^{n} P_{i}{ }^{2}}{N t}+m^{2}(x, y)= \\
m^{2}(x, y) \cdot\left(\frac{N t-1}{N t}\right)(6)
\end{gathered}
$$

If the total number of pixels, $\mathrm{Nt}$ is high, we can approximate $(\mathrm{C}-\mathrm{D})$ by $\mu^{2}(x, y)$. According to equation (5) if the input image is very dark or intensity values of pixels are low then the value of mean $\mu(x, y)$ at local windows is low so it drives that the difference between $\mathrm{C}$ and $\mathrm{D}$ is very small. But if the input image is bright or intensity values of pixels are high accordingly the value of mean $\mu(\mathrm{x}, \mathrm{y})$ is high and thus the difference between $\mathrm{C}$ and $\mathrm{D}$ is greater which means lowers the Binarization threshold value for NICK method. The value of Niblack factor $\mathrm{k}$ can vary from -0.1 to -0.2 depending upon the application requirement. For k close to -0.2 most of noise will be eliminated but characters can break or separate, while with values close to -0.1 , some noise pixels might remain but the text will be extracted clearly and unbroken. So, in our research approach we want to apply OCR application to detect license plate characters the appropriate value of $\mathrm{k}$ will be set at -0.1 .

NICK presents improved performance when compared to the other methods tested, and it performs better especially when the images have extremely low intensity variations and for bright local backgrounds in images. It's effective in compensation of low contrast and reducing the bad effects of noise because they are capable of adjusting themselves based on the local data information of the image. For example in some of license plate images with complex backgrounds around license plate area, it is hard to extract disjoint or ligatures character from shadow or bright illumination condition or snowy weather. Adaptive NICK thresholding typically takes a contrast enhancement grey scale input image and outputs a binary image, adaptive NICK filter will be apply as core thresholding filter in Multi-Scale thresholding in the ALPR system because in surrounding the license plate characters area there are enough foreground and background pixels in the local neighbourhood of each pixel.

A multi-scale Binarization framework is introduced by Moghadam [10], and followed by Bag [11]; this framework requires several Binarization on different scales. It is important to mention in this research the scales determined the local window size that we used for NICK adaptive thresholding methods. This enables us to explore local windows size scale to achieve the best thresholding results. All the foreground objects from previous scale (local window size) will be moved to new scale thresholding approaches; in this scenario we don't miss any object in the binary image as license plate character's candidate.

In this research scale is the size of sampling window that depends on the average size of character length in license plates on the image. Most of adaptive thresholding methods finally are limited to just one scale at the end. Also in some multi-scale methods the thresholding scale can be changed to detect very local features and structures based on different degradation sources and finally come up with one scale but the problem is just one scale cannot cover all of the degradation sources.

Most advantages of Multi-scale method is that it could be adapted for both types of degradation; interfering degradation and intensity degradation depends on which scale or window size you choose, therefore you can cover all the scales. The high scale size selected for interfering degradation recovery, typically, interfering degradation will be restored on high scales size to cover sufficient data from the original input image and interfering outlines. In compare, intensity degradation needs very small scales to deal with disjoint and broken characters in the license plate. So by multi-scale method the common solution for both types of degradation will be achieve.

This Method works in a Multi-Scale framework with an adaptive NICK thresholding core technique. The multiscale framework work to handle noises in degraded images.

Scales size will be determined based on prior information related to minimum and maximum size of local windows according to license plate characters approximation. Minimum and Maximum size of license plate character approximately determined based on image size. For input image of size $800 \times 600$ we select three scale 30, 60 and 120 pixels

In this method as Top- Down, first maximum size of local window as high scale 'Hs' will be select for adaptive NICK thresholding after Binarization the high scale 'Hs' divide by two and apply Binarization again till we reached minimum size of local window as low scale 'Ls' for Adaptive NICK thresholding. At each scale, only binarized foreground pixels that are connected to the foregrounds pixels of the previous scales are labelled as character otherwise, they will be rejected. Accordingly both type of image degradation will be improved and also connectivity of foreground object on different scale will be reminded, finally get an accurate binarized image Ib.

We apply this multi-scale processing to enhanced thresholding method against noise at different scale. After the final minimum label scaling size Ls, if any pixel is not classified then we mark them as background objects. This approach reduces the problem of character stroke discontinuities for degraded images.

\section{Image Thresholding Results}

Figure 2 shows grey scale input image on the snow condition, in figure 3 we zoom on the license plate area as it shows snow cover some portion of License plate character " $F$ " and it cause disconnection on first stage thresholding result on figure 4, by applying Multi-Scale Adaptive NICK thresholding method Character "F" connected and other character take improved shape. 
International Journal of Advanced Research in Computer and Communication Engineering Vol. 4, Issue 10, October 2015

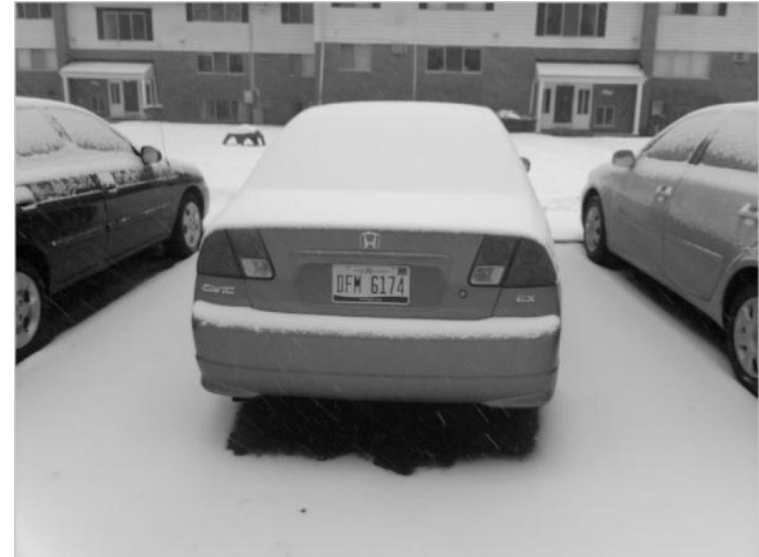

Fig. 2. Input Image

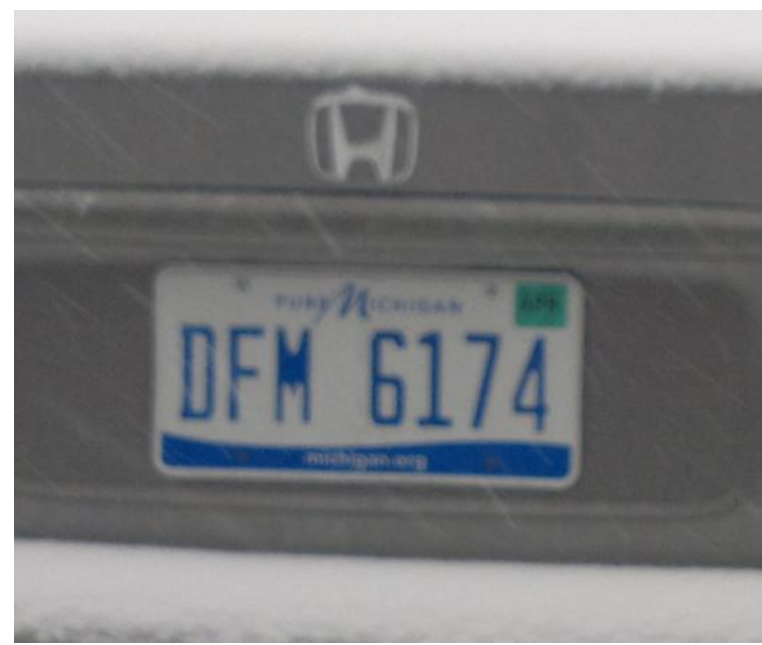

Fig. 3. License plate area on snow condition
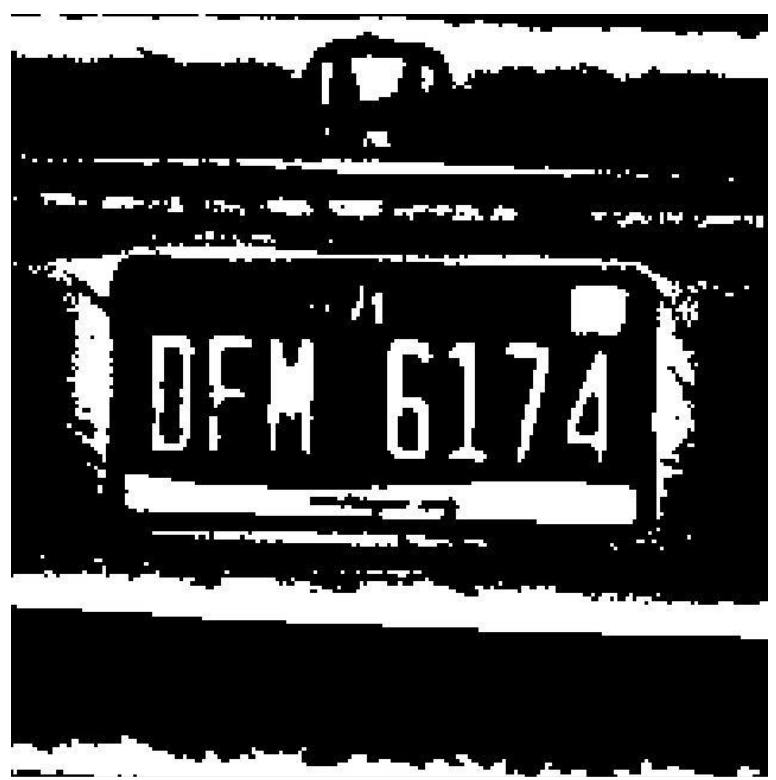

Fig. 4. First stage thresholding result

\section{CONCLUSION}

Multi-Scale Thresholding method presented as an improved thresholding method on ALPR system. In this method license plate under different illumination condition and weather condition will be detected. The accuracy of license plate characters detection step depends on thresholding method otherwise we might lose the characters candidates in the binary image. Results show this method work under various conditions of weather and illumination. This method could be adapted for both types of degradation; interfering degradation and intensity degradation, therefore it improves all types of degradation. The high scale size selected for interfering degradation recovery, interfering degradation will be restored on high scales size to cover sufficient data from the original input image. In compare, intensity degradation needs very small scales to deal with disjoint and broken characters in the license plate. As a result in this method we don't miss any object in the binary image as license plate character's candidate.

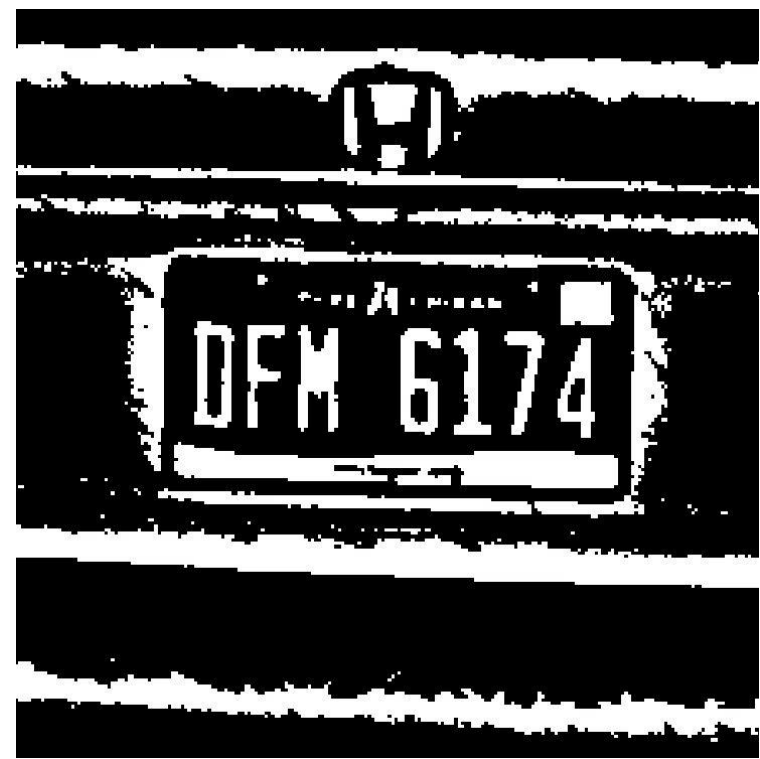

Fig. 5. Final Multi-Scale Adaptive NICK Thresholding result

\section{REFERENCES}

[1] Shan Du, Member, IEEE, Mahmoud Ibrahim, Mohamed Shehata, Senior Member, IEEE, and Wael Badawy, Senior Member, IEEE," Automatic License Plate Recognition (ALPR): A State-of-the-Art Review", IEEE TRANSACTIONS ON CIRCUITS AND SYSTEMS FOR VIDEO TECHNOLOGY, VOL. 23, NO. 2, FEBRUARY 2013

[2] Christos-Nikolaos E. Anagnostopoulos, Ioannis D. Psoroulas, Vassili Loumos and Eleftherios Kayafas, "License Plate Recognition From Still Images and Video Sequences: A Survey", IEEE TRANSACTIONS ON INTELLIGENT TRANSPORTATION SYSTEMS, VOL. 9, NO. 3, SEPTEMBER 2008

[3] Mehmet Sezgin, Bülent Sankur,*, Mehmet Sezginb IMAGE THRESGOLDING TECHNIQUES: A SURVEY OVER CATEGORIES', Journal of Electronic Imaging 13(1), 146-165 (January 2004)

[4] NOBUYUKI OTSU, "A Tlreshold Selection Method from GrayLevel Histograms", IEEE TRANSACTIONS ON SYSTREMS, MAN, AND CYBERNETICS, VOL. SMC-9, NO. 1, JANUARY 1979

[5] Prasanna K. Sahooa; Gurdial Arorab, "A thresholding method based on two-dimensional Renyi's entropy", Elsevier Ltd, Pattern Recognition 37 (2004) 1149 - 1161

[6] F. Deravi, S.K. Pal, "Grey level thresholding using second-order statistics", Pattern Recognition Letters, 1 (1983) 417-422.

[7] Niblack, W.: An Introduction to Digital Image Processing. Englewood Cliffs, N. J., Prentice Hall (1986) 115-116 
[8] J. Bernsen, Dynamic Thresholding of Grey level Images, ICPR'86: Proc. Int. Conf. on Pattern Recognition, Berlin, Germany, 1986, pp:1251-1255

[9] Sauvola, J., Pietikainen, M.: “Adaptive Document Image Binarization", Pattern recognition 33(2000), 225-236

[10] Reza Farrahi Moghaddam, Mohamed Cheriet, "A multi-scale framework for adaptive binarization of degraded document images", Pattern Recognition 43 (2010) 2186-2198.

[11] Soumen Bag, Partha Bhowmick, "Adaptive-interpolative binarization with stroke preservation for restoration of faint characters in degraded documents", J. Vis. Commun. Image R. 31 (2015) 266-281

[12] Derek Bradley, Gerhard Roth, "Adaptive Thresholding Using the Integral Image", Journal of Graphics, GPU, and Game Tools Volume 12, Issue 2, 2007

[13] J. Padhye, V. Firoiu, and D. Towsley, "A stochastic model of TCP Reno congestion avoidance and control," Univ. of Massachusetts, Amherst, MA, CMPSCI Tech. Rep. 99-02, 1999.

[14] Wireless LAN Medium Access Control (MAC) and Physical Layer (PHY) Specification, IEEE Std. 802.11, 1997.

[15] M. M. Mokji, S.A.R. Abu Bakar, Adaptive Thresholding Based On Co-Occurrence Matrix Edge Information, JOURNAL OF COMPUTERS, VOL. 2, NO. 8, OCTOBER 2007

\section{BIOGRAPHIES}

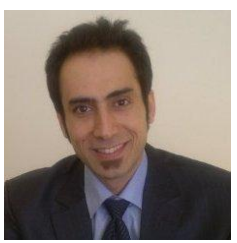

Roho llah Mazrae Khoshki, $\mathrm{PhD}$ student in Oakland University, Rochester, MI, USA, in Embedded System Electrical Eng. He investigated and designed of Interferometric Fiber Optic Gyroscope (IFOG) as his Master Thesis. He is interested in Embedded Systems, FPGAs and Microcontrollers, Robotics and control, Image processing and Optoelectronic in research and practical applications. More information on him is at: https://www.linkedin.com/pub/rohollah-mazrae-khoshki/53/14/973

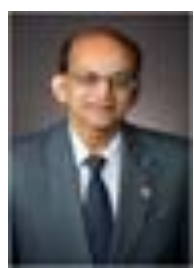

Dr. Subramaniam Ganesan is a Professor of Electrical and Computer Engineering, Oakland University, Rochester, MI, 48309, USA. He has over 25 years of teaching and research experience in Digital systems. He served as the chair of the CSE department from 1991 to 98 . He is with Electrical and Computer Engineering Department since 2008. He received his masters and Ph.D. from Indian Institute of Science (IISc) Bangalore, India. He worked at National Aeronautical Laboratory (NAL) India, Ruhr University, Germany, Concordia University Canada, and Western Michigan University before joining Oakland University in 1986. More information on him is at: www.secs.oakland.edu/ ganesan 